\title{
INVARIANT MEASURES AND RADON-NIKODYM DERIVATIVES
}

\author{
BY \\ DOROTHY MAHARAM( $\left.{ }^{1}\right)$
}

\section{0 . Introduction.}

0.1. Suppose we are given a nonsingular measurable one-one transformation $T$ of a measure space $(X, m)$ onto itself. The main object of this paper is to answer the question: Under what conditions will there exist a measure $\mu$ (equivalent to $m$ ) which is invariant under $T$ (that is, with respect to which $T$ becomes measurepreserving $\left({ }^{2}\right)$ ? We shall see that this question can be answered, in principle, if one merely knows the sequence of Radon-Nikodym derivatives $\omega_{n}(x)=d m\left(T^{n} x\right) / d m(x)$ $(n=1,2, \ldots)$. We obtain necessary and sufficient conditions for the existence of an invariant measure in terms of these derivatives (see Theorem 7, in 5.1 below). We also obtain a construction for an invariant measure, when one exists; and we discuss a few other questions which arise in the course of the investigation.

All measures considered are to be $\sigma$-finite and equivalent to $m$; and, throughout almost all the paper, we make the restriction that the given measure $m$ is finite. This involves no real loss of generality; and in 6.1 we discuss briefly the effect of eliminating this restriction. But the finiteness of $m$ does enable most of our results to be stated much more simply.

In the case of a finite invariant measure, the situation is relatively simple; in Theorem 1 (3.2) a number of criteria, not all of them new, are given. Perhaps the simplest of them (essentially known) is that a finite invariant measure exists if and only if the $(C, 1)$ limit $l(x)$ of the sequence $\left\{\omega_{n}(x)\right\}$ exists and is positive a.e.; further, if this condition is fulfilled, $\int l d m$ gives a finite invariant measure. The case of an infinite invariant measure is decidedly more complicated. The main idea is to average the sequence $\left\{\omega_{n}(x)\right\}$ in a more delicate way, by introducing the notion of a "density average". For any sufficiently "smooth" nonnegative sequence $\{\xi(n)\}$ and for all $\alpha, \beta \geqq 0$, we consider the relative asymptotic density of the $n$ 's for which $\xi(n) \geqq \alpha$ as compared with those for which $\xi(n) \geqq \beta$, and integrate this with respect to $\alpha$ to get the "density average relative to $\beta$ ". It turns out that, when applied to the sequence $\left\{\omega_{n}(x)\right\}$ with $\beta=0$, this process produces the $(C, 1)$ limit and thus gives a criterion and a simple construction for a finite invariant measure.

Received by the editors September 16, 1966 and, in revised form, August 14, 1967.

${ }^{(1)}$ This research was completed while the author held a National Science Foundation Senior Post-Doctoral Fellowship.

$\left({ }^{2}\right)$ For some other answers to this question, see the survey in [9, pp. 93-100], and also [1], [2], [4], [5]. 
Applied with $\beta>0$, it gives a necessary and sufficient condition for the existence of a possibly infinite invariant measure (Theorem $7,5.1$ ). It also gives a construction for an invariant measure when there is one; but, remarkably, this construction does not consist merely in integrating the $\beta$-average. Instead, the finiteness of the $\beta$-average is used to show that a quite different limiting process now gives the desired measure. The criteria and construction for the invariant measure are reasonably "effective", and the author hopes that they may even be practicable in some simple cases; the main difficulty in applying the theory is that the functions $\omega_{n}$ themselves are usually rather complicated.

0.2. In more detail, we proceed as follows. In $\$ 1$, we introduce the notions of "density sequence" and "density average", and derive some of their basic properties. After recalling (\$2) some well-known criteria for invariant measures, we deal with the case of a finite invariant measure in $\$ 3$. In $\$ 4$, after disposing of the dissipative case, we prove (Theorem $4,4.8$ ) that our main condition for the existence of an invariant measure is necessary; this we derive from a criterion for a function $f$ to be "invariantly $\sigma$-summable" (that is, summable on each member of a sequence of invariant sets whose union is $X$ ) which may be of independent interest (Theorem $3,4.4)$. The proof of sufficiency is postponed while we consider some results which follow from the methods used to prove necessity. One such result (4.5) is a sharpening of the usual ergodic theorem: under the usual hypotheses, the $(C, 1)$ limit of the sequence $\left\{f\left(T^{n} x\right)\right\}$ is "stable" in the sense that it is not affected by the deletion of any subsequence of its terms for which the $n$ 's have asymptotic density 0 . Another (4.6) gives a formula for the density averages of the sequence $\left\{f\left(T^{\tilde{n}} x\right)\right\}$ under certain conditions (principally that $T$ be measure-preserving and ergodic). In the remainder of $\S 4$ we discuss the limiting behavior of the density function of the sequence $\left\{\omega_{n}(x)\right\}$ (Theorems 5,6 ).

The converse of Theorem 4 -that the necessary condition for the existence of an invariant measure is also sufficient-is proved in $\$ 5$ (Theorem 7). Finally, in $\S 6$ we first restate some sample results so as to free them from the assumption $m(X)$ $<\infty$ (Theorem 8), and then apply Theorem 7 to show that (roughly speaking) a direct sum of measure spaces with invariant summands admits an invariant measure if and only if almost all the summands do.

I am greatly indebted to Y. Ito for a major simplification of the proof of sufficiency (Theorem 7). His ingenious improvement of my original construction made it unnecessary to proceed (at considerable length) from a special case to the general one. I am also grateful to A. H. Stone for many helpful discussions; in particular, the treatment of density sequences in $\S 1$ has benefitted from his suggestions.

0.3. Notation. A measure space $(X, \mathscr{B}, m)$ is usually denoted by $(X, m)$, or sometimes $X$ for short; the measure is always assumed $\sigma$-finite, and we usually suppose (for simplicity, and without loss of generality) that the field $\mathscr{B}$ of measurable sets contains all subsets of null sets. A "nonsingular measurable transformation" $T$ of the measure space $(X, m)$ is a 1-1 mapping of $X$ onto $X$ such that (for all 
$A \subset X) T A$ is measurable if and only if $A$ is measurable, and null if and only if $A$ is null. A subset $A$ of $X$ is "invariant" under $T$ provided that $T A=A$. Clearly every null set is contained in an invariant null set, and we reserve the right to discard invariant null sets without further notice.

Functions on $X$ are understood to be real-valued, unless the contrary is stated. A function $f$ is "summable" (with respect to $m$ ) on $X$ provided $\int_{X}|f| d m<\infty$. Given a nonsingular measurable transformation $T$ of $(X, m)$, the Radon-Nikodym derivatives $\omega_{i}(x)(i=0, \pm 1, \pm 2, \ldots)$ are defined a.e. so that

$$
\int_{X} f\left(T^{i} x\right) \omega_{i}(x) d m(x)=\int_{X} f(x) d m(x)
$$

whenever $f$ is summable or nonnegative; of course $\omega_{0}=1$, and we write $\omega_{1}$ as $\omega$. $\mathscr{N}$ denotes the set of nonnegative integers; and we are interested in $\omega_{n}$ mainly for $n \in \mathscr{N}$. We write $\lim \sup _{n \rightarrow \infty} \omega_{n}(x)=\omega^{*}(x), \lim _{\inf _{n \rightarrow \infty}} \omega_{n}(x)=\omega_{*}(x)$. We adopt the usual conventions that $\alpha / 0=\infty$ for $\alpha>0$ and $\alpha / \infty=0$ for finite $\alpha$, and the unusual ones that $0 / 0=0$ and $\infty / \infty=1$; however, $0 . \infty$ is not defined.

To avoid repetition, we assume the following "standing hypothesis" throughout, except where the contrary is stated:

$T$ is a nonsingular measurable transformation of the measure space $(X, m)$, and $m X<\infty$.

\section{Density sequences and averages.}

1.1. Let $\xi=\{\xi(n) \mid n \in \mathscr{N}\}$, where $\mathscr{N}=\{0,1,2, \ldots\}$, be a sequence of nonnegative numbers; we shall be interested mainly in the case in which $\xi(n)=\omega_{n}(x)$, $x$ being a "general" point of $X$, but it is convenient to consider a more general situation $\left({ }^{3}\right)$. For each $n \in \mathscr{N}$ and (finite) $\alpha, \beta \geqq 0$, write

$$
\delta_{n}(\alpha, \beta)=\frac{\text { number of } i \text { 's } \in \mathscr{N} \text { such that } i \leqq n \text { and } \xi(i) \geqq \alpha}{\text { number of } i \text { 's } \in \mathscr{N} \text { such that } i \leqq n \text { and } \xi(i) \geqq \beta}
$$

(with the conventions $0 / 0=0, n / 0=\infty$ for $n=1,2, \ldots$, as in $\S 0.3$ ). If $\lim _{n \rightarrow \infty} \delta_{n}(\alpha, \beta)$ exists (possibly infinite) for all $\alpha, \beta \geqq 0$, we denote this limit by $\delta(\alpha, \beta ; \xi)$ or $\delta(\alpha, \beta)$ for short, and say that $\xi$ is a density sequence, with relative density function $\delta$. It is easy to see that every monotone sequence $\xi$ is a density sequence; some other examples are mentioned later.

1.2. From now on, we assume that $\xi$ is a given density sequence (of nonnegative numbers). We have the following easily verified properties:

(1) $\delta(\beta, \alpha)=1 / \delta(\alpha, \beta)$, unless $\xi(n)<\min \{\alpha, \beta\}$ for all $n \in \mathscr{N}$ (in which case $\delta(\beta, \alpha)=0=\delta(\alpha, \beta))$.

(2) $\delta(\alpha, \gamma)=\delta(\alpha, \beta) \cdot \delta(\beta, \gamma)$ unless the right side is $0 \cdot \infty$ or $\infty \cdot 0$.

(3) If $0 \leqq \alpha \leqq \alpha^{\prime}$ and $0 \leqq \beta^{\prime} \leqq \beta$ then $\delta(\alpha, \beta) \geqq \delta\left(\alpha^{\prime}, \beta^{\prime}\right) \geqq 0$.

(4) $\delta(\alpha, \alpha)=1$ unless $\xi(n)<\alpha$ for all $n \in \mathscr{N}$ (in which case $\delta(\alpha, \alpha)=0$ ).

${ }^{3}$ ) We could also consider sequences of arbitrary real numbers, but the restriction to nonnegative numbers simplifies matters somewhat and suffices for the applications here. A related (but different) type of sequence has been introduced by Sirikov [14]. 
Of course, $\delta(\alpha, 0)$ is just the asymptotic density of the set of integers $i$ for which $\xi(i) \geqq \alpha$. In particular,

(5) $\delta(\alpha, 0) \leqq 1$.

From (3), for each fixed $\beta \geqq 0, \delta(\alpha, \beta)$ is a decreasing and hence measurable function of $\alpha$. We define the "density average of $\xi$ relative to $\beta$ " by:

(6) $A_{\beta}=A_{\beta}(\xi)=\int_{0}^{\infty}, \delta(\alpha, \beta) d \alpha$;

thus $0 \leqq A_{\beta} \leqq \infty$, and in fact one easily sees

(7) $A_{\beta} \geqq \beta$ for all $\beta \geqq 0$, unless $\xi(n)=0$ for all $n \in \mathscr{N}$.

It follows easily from (2) that

(8) $A_{\gamma}=\delta(\beta, \gamma) A_{\beta}$ if $0<\delta(\beta, \gamma)<\infty$,

and from (3) that

(9) $A_{\beta}$ is an increasing function of $\beta$.

1.3. Further properties of $A_{\beta}$. We shall later need:

(1) For every density sequence $\xi$, other than the zero sequence, $\int_{0}^{\infty}\left(1 / A_{\beta}\right) d \beta=0$ or $1 ; 0$ if $A_{\gamma}=\infty$ for all $\gamma>0,1$ otherwise $\left({ }^{4}\right)$.

We may assume $\xi\left(n_{0}\right)>0$ for some $n_{0} \in \mathscr{N}$, and that $A_{\gamma}<\infty$ for some $\gamma>0$; because of 1.2(9) we may suppose $\gamma<\xi\left(n_{0}\right)$. Then $\delta(\alpha, \gamma)<\infty$ for all $\alpha>0$, because it is monotone in $\alpha$ and has a finite integral. Put $b=\sup \{\alpha \mid \alpha \geqq 0, \delta(\alpha, \gamma)>0\}$; from $1.2(4), 0<\gamma \leqq b \leqq \infty$. We readily check, from $1.2(2)$ and $1.2(1)$, that if $0<\dot{\alpha}, \beta<b$ we have $\delta(\alpha, \beta)=\delta(\alpha, \gamma) \delta(\gamma, \beta)$, where all these quantities are positive and finite; but that if $0<\alpha<b<\beta$ then $\delta(\alpha, \beta)=\infty$ and $\delta(\beta, \alpha)=0$. (The only point requiring care is that $0 / 0$ situations must be avoided, and this is why we make $\gamma<\xi\left(n_{0}\right)$.) Thus $A_{\beta}=\infty$ if $\beta>b$, while if $0<\beta<b$ we have (1.2(8))

Hence

$$
A_{\beta}=\int_{0}^{b} \delta(\alpha, \beta) d \alpha=\delta(\gamma, \beta) A_{\gamma}
$$

$$
\int_{0}^{\infty}\left(1 / A_{\beta}\right) d \beta=\left(1 / A_{\gamma}\right) \int_{0}^{\infty} \delta(\beta, \gamma) d \beta=1
$$

Since the integral in (1) converges in either case, we have

(2) For every density sequence $\xi$, other than the zero sequence, $A_{\beta}$ is not $\mathcal{O}(\beta)$ when $\beta \rightarrow \infty$.

It also follows that, even if $\lim _{\beta \rightarrow 0+} A_{\beta}=0$-which need not be the case-we cannot have $A_{\beta}=\mathcal{O}(\beta)$ for $\beta \rightarrow 0+$ (compare 1.2(7)).

(3) If $\lim _{n \rightarrow \infty} \xi(n)=0$ then $\xi$ is a density sequence; and, unless $\xi$ is the zero sequence, $\sum_{0}^{\infty} \xi(n)$ converges if and only if $A_{\gamma}<\infty$ for some $\gamma>0$; that is (from (1)) if and only if $\int_{0}^{\infty}\left(1 / A_{\beta}\right) d \beta=1$.

It is easily verified that $\xi$ is a density sequence; moreover we have for $\alpha, \beta>0$ that

$$
\begin{aligned}
\delta(\alpha, \beta)= & \delta_{n}(\alpha, \beta) \text { for all large enough } n, \\
= & \frac{\sum_{i}\{1 \text { if } \xi(i) \geqq \alpha, 0 \text { otherwise }\}}{\sum_{i}\{1 \text { if } \xi(i) \geqq \beta, 0 \text { otherwise }\}},
\end{aligned}
$$

$\left(^{4}\right)$ The "zero sequence" is the one for which $\xi(n)=0$ for all $n \in \mathscr{N}$. 
where both numerator and denominator are finite sums, and the denominator is nonzero if $\beta$ is small enough. Integrating, we get $A_{\beta}=(1 / c) \sum_{i} \xi(i)$ where $c$ is the above denominator, and the result follows.

We shall later generalize (3) considerably (Theorem 4, Corollary 2, 4.5).

(4) If $0<A_{0}<\infty$, then $0<A_{\gamma}<\infty$ for all small enough positive $\gamma$.

For we must have $\delta(\beta, 0)>0$ for some $\beta>0$ (else $A_{0}=0$ ); and then, if $0<\gamma<\beta$, we have that $1 \leqq \delta(0, \gamma)<\infty$ and $A_{\gamma}=\delta(0, \gamma) A_{0}$ from 1.2(8). (The converse of (4) is false; $A_{0}$ may be 0 .)

Finally, by way of illustration, we remark that if the sequence $\xi$ is uniformly distributed in $[0,1]$ then it is a density sequence, and $A_{\beta}=1 / 2(1-\beta)$ for $0 \leqq \beta<1$, $\infty$ for $\beta \geqq 1$.

1.4. $A_{0}$ and $(C, 1)$ limits. In this section it is convenient to consider a notion more general than that of a density sequence. We say that a sequence $\xi$ of nonnegative real numbers is a "weak density sequence" if it satisfies the requirements for a density sequence when $\beta=0$; that is, $\delta(\alpha, 0)=\lim _{n \rightarrow \infty} \delta_{n}(\alpha, 0)$ exists (perhaps infinite) for all $\alpha \geqq 0$. Thus $A_{0}(\xi)$ is still defined (by $1.2(6)$ ). We clearly have:

(1) If two nonnegative sequences $\xi, \eta$ agree except on a set of integers of asymptotic density 0 , and if $\xi$ is a weak density sequence, then so is $\eta$; and they have the same density function $\delta(\alpha, 0)$, and consequently the same $A_{0}$.

Next we show:

(2) If $\xi$ is a bounded weak density sequence, $\lim _{n \rightarrow \infty}(1 /(n+1))(\xi(0)+\xi(1)+\cdots$ $+\xi(n))$ exists and equals $A_{0}(\xi)$.

Sketch of proof. Say $0 \leqq \xi(n)<M$ for all $n \in \mathscr{N}$. Divide the interval $[0, M)$ into consecutive small intervals $\left[x_{i}, x_{i+1}\right)(i=1,2, \ldots, k)$ and consider $\xi(0)+\xi(1)$ $+\cdots+\xi(n)$ for large $n$. The number of summands $\xi(j)$ falling in $\left[x_{i}, x_{i+1}\right)$ will be approximately $(n+1)\left(\delta\left(x_{i}, 0\right)-\delta\left(x_{i+1}, 0\right)\right)$; hence $(1 /(n+1))(\xi(0)+\cdots+\xi(n))$ is approximately

$$
\sum_{i} x_{i}\left(\delta\left(x_{i}, 0\right)-\delta\left(x_{i+1}, 0\right)\right)=\sum_{i} \delta\left(x_{i}, 0\right)\left(x_{i}-x_{i+1}\right),
$$

a Riemann sum for the integral $A_{0}(\xi)$.

We deduce from this:

(3) For an arbitrary weak density sequence $\xi$,

$$
\liminf _{n \rightarrow \infty} \frac{\xi(0)+\cdots+\xi(n)}{n+1} \geqq A_{0}(\xi) .
$$

For, fixing a positive number $k$, put $\xi^{k}(i)=\min \{\xi(i), k\}(i \in \mathscr{N})$. Note that $\xi^{k}$ is also a weak density sequence, and that $\delta\left(\alpha, 0 ; \xi^{k}\right)=\delta(\alpha, 0 ; \xi)$ if $\alpha \leqq k$, and is 0 if $\alpha>k$. Now $\xi(i) \geqq \xi^{k}(i)$, so

$$
\liminf _{n \rightarrow \infty}(\xi(0)+\cdots+\xi(n)) /(n+1) \geqq \liminf _{n \rightarrow \infty}\left(\xi^{k}(0)+\cdots+\xi^{k}(n)\right) /(n+1)=A_{0}\left(\xi^{k}\right)
$$

by (2), $=\int_{0}^{\infty} \delta\left(\alpha, 0 ; \xi^{k}\right) d \alpha=\int_{0}^{k} \delta(\alpha, 0 ; \xi) d \alpha$; and on making $k \rightarrow \infty$ we obtain (3). 
It should be remarked that for unbounded sequences $\xi$, (2) fails in general, even when $\xi$ is a density sequence whose $(C, 1)$ limit exists (for instance, put $\xi(n)=0$ unless $n=i^{2}$, and $\left.\xi\left(i^{2}\right)=i\right)$. This is essentially because altering $\xi$ on a set of integers of asymptotic density 0 will not alter $A_{0}(\xi)$, by (1). We have, however, for an arbitrary weak density sequence $\xi$, the following observation of A. H. Stone:

(4) $A_{0}(\xi)$ is the smallest real number $a$ with the following property: by altering $\xi$ on a set of integers of asymptotic density 0 , we can obtain a sequence (of nonnegative numbers) with $(C, 1)$ limit a.

In fact, if $\eta$ is obtained by such an alteration, we have by (1) that $A_{0}(\xi)=A_{0}(\eta)$, which $\leqq a$ by (3). On the other hand, we can obtain such an $\eta$ as follows. We may assume $A_{0}(\xi) \neq \infty$ (otherwise put $\eta=\xi$ ). For $k=1,2, \ldots$, write

$$
\mathscr{N}_{k}=\{n \mid n \in \mathscr{N}, \xi(n) \geqq k\} ;
$$

then $\mathscr{N}_{k}$ has asymptotic density $\delta(k, 0 ; \xi)$, and one readily verifies that this cannot exceed $A_{0}(\xi) / k$. Define $\eta^{k}(n)=\xi(n)$ if $n \in \mathscr{N}-\mathscr{N}_{k}, 0$ if $n \in \mathscr{N}_{k}$; one checks that $\eta^{k}$ is also a weak density sequence, so that (by (2))

$$
\lim _{n \rightarrow \infty}\left(\eta^{k}(0)+\cdots+\eta^{k}(n)\right) /(n+1)=A_{0}\left(\eta^{k}\right) \leqq A_{0}(\xi)
$$

Choosing $n_{1}, n_{2}, \ldots$ to be a sufficiently rapidly increasing sequence, we define $\eta(n)=\eta^{k}(n)$ where $k=k(n)$ is determined so that $n_{k}<n \leqq n_{k+1}$ (and $\eta(n)=0$ if $\left.n \leqq n_{1}\right)$. Thus $\eta(n)$ is either $\xi(n)$ or 0 ; we put $\mathscr{M}=\{n \mid \eta(n) \neq \xi(n)\}$. Since $\mathscr{N} \supset \mathscr{N}_{1} \supset \mathscr{N}_{2} \supset \cdots$, we have $\mathscr{M} \subset\left\{0,1, \ldots, n_{k}\right\} \cup \mathscr{N}_{k}(k=1,2, \ldots)$, from which it is easy to see that $\mathscr{M}$ has asymptotic density 0 . Also $\eta^{1}(n) \leqq \eta^{2}(n) \leqq \cdots(n \in \mathscr{N})$, so that if $n \leqq n_{k+1}$ we have $\eta(n) \leqq \eta^{k}(n)$. Hence, if $n_{k}<n \leqq n_{k+1}$, we have

$$
(\eta(0)+\cdots+\eta(n)) /(n+1) \leqq\left(\eta^{k}(0)+\cdots+\eta^{k}(n)\right) /(n+1) \leqq A_{0}(\xi)+1 / k
$$

if $n_{k}$ is large enough. Combining this with (3), we have

$$
\lim _{n \rightarrow \infty}(\eta(0)+\cdots+\eta(n)) /(n+1)=A_{0}(\xi) .
$$

We shall later see (3.9(4)) that when $\xi(n)=\omega_{n}(x)$ there is equality in (3), and that moreover $A_{0}$ for this sequence is closely related to the existence of a finite invariant measure (see Theorem 1, 3.1). This motivates the adoption of $A_{\beta}$, for $\beta>0$, as a generalized averaging process which we shall see (Theorem $7,5.1$ ) is related to the existence of a possibly infinite invariant measure. Of course, if $0<\delta(\beta, 0)<\infty$, it follows from $1.2(1)$ and $1.2(8)$ that $A_{\beta}=\delta(0, \beta) A_{0}$, so that $A_{\beta}$ would give nothing new; but in the application $\delta(\beta, 0)$ will usually be 0 (see Theorem $6,4.9$ ).

1.5. Some density sequences of importance to us, and some of their properties, are provided by Theorems 1 and 2 of [13], which we now restate in a slightly extended form. The standing hypothesis $(0.3)$ applies to both of them, except that we do not require $m(X)$ to be finite. 
TheOREM A. Suppose $T$ is measure-preserving. Let $f$ be a positive measurable real-valued function on $X$ such that for all $\gamma \geqq 0, m\{x \mid f(x) \geqq \gamma\}<\infty$. Then there exists an invariant null set $N$ such that, for all $x \in X-N$, the sequence $\xi=\left\{f\left(T^{n} x\right) \mid n \in \mathscr{N}\right\}$ is a density sequence. Further, denoting $\delta(\alpha, \beta ; \xi)$ by $D(\alpha, \beta ; x)$ (where $x \in X-N$ and $\alpha, \beta \geqq 0$ ) we have that $D$ is fully measurable $\left({ }^{5}\right)$.

THEOREM B. Suppose further that $T$ is incompressible, and write

$$
F(x)=\limsup _{n \rightarrow \infty} f\left(T^{n} x\right)
$$

Then $F$ is measurable, invariant and positive on $X-N$; further,

(i) if $x \in X-N$ and $0<\alpha, \beta<F(x)$, then $0<D(\alpha, \beta ; x)<\infty$;

(ii) if $x \in X-N$ and $\alpha, \beta \geqq 0$, then $D(\alpha, \beta ; x)=D(\alpha, \beta ; T x)$, and each of the sets $\left\{n \mid n \in \mathscr{N}, f\left(T^{n} x\right) \geqq \alpha\right\}$ is either infinite or empty.

The main difference between the above theorems and the theorems in [13] is that in [13] the restriction was imposed that $\alpha \geqq \beta$. However, it is a routine matter (using $1.2(1))$ to check that if the density $D(\alpha, \beta ; x)$ exists and has the above properties when $\alpha \geqq \beta \geqq 0$, it also exists and has these properties when $0 \leqq \alpha<\beta$. We have made two other minor alterations: (a) the statement in Theorem $\mathrm{A}$ that $D$ is fully measurable has been transferred from [13, Theorem 2]; the hypothesis there that $T$ is incompressible was not needed for this statement, as was pointed out in the proof of [13, Theorem 2]; (b) in [13], the relative densities $D(\alpha, \beta ; x)$ were defined using the set of positive integers, rather than (as here) the nonnegative integers; of course, this makes no real difference.

For the sequence $\xi=\left\{f\left(T^{i} x\right) \mid i=0,1,2, \ldots\right\}$ arising here, we write $A_{\beta}(\xi)$ as $J_{B}(x)$; that is,

$$
J_{\beta}(x)=\int_{0}^{\infty} D(\alpha, \beta ; x) d \alpha \quad(\text { for } \beta \geqq 0 \text { and } x \in X-N) .
$$

Note that $J_{\beta}(T x)=J_{\beta}(x)$, from Theorem B (ii).

1.6. Two further theorems of [13] concern a density sequence which will be even more important for us than the preceding one. We restate them here, slightly modified (in the same way as the two previous theorems were modified). We make the standing hypothesis, including the requirement that $m(X)<\infty$. (Note that $T$ need not be measure-preserving, however.)

THEOREM C. There exists an invariant null set $N$ such that, for all $x \in X-N$, the sequence $\xi=\left\{\omega_{n}(x) \mid n \in \mathscr{N}\right\}$ is a density sequence. Further, denoting $\delta(\alpha, \beta ; \xi)$ by $d(\alpha, \beta ; x)$ (where $x \in X-N$ and $\alpha, \beta \geqq 0$ ), we have that $d$ is fully measurable. others.

$\left(^{5}\right)$ That is, if some (or none) of $\alpha, \beta, x$ are fixed, $D(\alpha, \beta ; x)$ is a measurable function of the 
THEOREM D. Suppose further that $T$ is incompressible. Then $\omega^{*}=\lim \sup _{n \rightarrow \infty} \omega_{n}$ is measurable and positive on $X-N$; further,

(i) if $x \in X-N$ and $0<\alpha, \beta<\omega^{*}(x)$, then $0<d(\alpha, \beta ; x)<\infty$;

(ii) if $x \in X-N$ and $\alpha, \beta \geqq 0$, then $d(\alpha \omega(x), \beta \omega(x) ; x)=d(\alpha, \beta ; T x)$, and each of the sets $\left\{n \mid n \in \mathscr{N}, \omega_{n}(x) \geqq \alpha\right\}$ is either infinite or empty $\left({ }^{6}\right)$.

For the sequence $\xi=\left\{\omega_{n}(x) \mid n \in \mathscr{N}\right\}$ arising here, we write $A_{\beta}(\xi)$ as $I_{\beta}(x)$; that is, $I_{\beta}(x)=\int_{0}^{\infty} d(\alpha, \beta ; x) d \alpha$ (where $x \in X-N$ and $\beta \geqq 0$ ). Note that $I_{\beta}(x)$ is, for fixed $x$, an increasing function of $\beta$ (from 1.2(9)). Again, from Theorem D (ii) we obtain:

(1) If $T$ is incompressible, $I_{\beta \omega(x)}(x)=\omega(x) I_{\beta}(T x)$ a.e., for all $\beta \geqq 0$; and by induction over $n$ (using (2.1(1) below) one deduces from (1) that:

(2) If $T$ is incompressible, $I_{\beta \omega_{n}(x)}(x)=\omega_{n}(x) I_{\beta}\left(T^{n} x\right)$ a.e., for all $\beta \geqq 0$ and $n=1,2, \ldots$, a result which will be useful later.

1.7. The requirement, in Theorems $\mathrm{B}$ and $\mathrm{D}$, that $T$ be incompressible does not involve much loss of generality, because in any case we may express $X$ (uniquely, to within null sets) as the union of disjoint invariant sets $X_{1}, X_{2}$ such that $T \mid X_{1}$ is incompressible and $T \mid X_{2}$ is purely dissipative. From Theorem $\mathrm{D}(\mathrm{i}), \omega^{*}(x)>0$ a.e. on $X_{1}$, and it is well known that $\sum_{-\infty}^{\infty} \omega_{n}(x)<\infty$ a.e. on $X_{2}\left({ }^{7}\right)$; thus

(1) $X_{1}=\left\{x \mid \omega^{*}(x)>0\right\}, X_{2}=\left\{x \mid \omega^{*}(x)=0\right\}$.

From this we deduce a fact which will be useful later:

(2) If $d(\alpha, 0 ; x)>0$ for some $\alpha>0$, then $\omega^{*}(x)>0$, and $d\left(\alpha^{\prime}, 0 ; x\right)>0$ for every $\alpha^{\prime}$ such that $0 \leqq \alpha^{\prime}<\omega^{*}(x)$.

For one easily sees that $\omega^{*}(x) \geqq \alpha>0$; thus $x \in X_{1}$. If $0<\alpha^{\prime}<\omega^{*}(x)$, we fix a positive $\beta<\alpha$; then $(1.2(3)) d(\beta, 0 ; x) \geqq d(\alpha, 0 ; x)>0$, and (from Theorem $\mathrm{D}(\mathrm{i}$ ) applied to $\left.X_{1}\right) 0<d\left(\alpha^{\prime}, \beta ; x\right)<\infty$. Thus $(1.2(2)) d\left(\alpha^{\prime}, 0 ; x\right)=d\left(\alpha^{\prime}, \beta ; x\right) d(\beta, 0 ; x)>0$. Finally, if $\alpha^{\prime}=0$ we have $d(0,0 ; x)=1$ trivially.

We shall also need the following immediate consequence of Theorem $\mathrm{D}$ (applied to $X_{1}$ ) and $1.2(8)$ :

(3) If $0<\beta, \gamma<\omega^{*}(x)$, then $I_{\gamma}(x)=d(\beta, \gamma ; x) I_{\beta}(x)$; and in particular if $I_{\beta}(x)$ is positive, or finite, then so is $I_{\gamma}(x)$.

\section{Lemmas on invariant measures.}

2.1. We collect here some well-known (and easily verified) results which will be useful to us. We make the standing hypothesis (though in fact most of the results do not need the finiteness of $m$ ). First we have

$$
\omega_{i+1}(x)=\omega_{i}(T x) \omega(x) \text { a.e. } \quad(i=0, \pm 1, \pm 2, \ldots) .
$$

From this, by an easy induction,

$$
\omega_{n}(x)=\omega(x) \omega(T x) \cdots \omega\left(T^{n-1} x\right) \text { a.e. } \quad(n=0,1,2, \ldots) .
$$

$\left({ }^{6}\right)$ In [13, Theorem 4] it was inadvertently stated that $\omega^{*}$ is invariant under $T$; actually $\omega^{*}(T x)=\omega^{*}(x) / \omega(x)$ (see (2.2) below).

$\left({ }^{7}\right)$ See for instance [12, end of 8.2$]$ for a proof. 
Next, because of the finiteness of $m X$, we have

$$
\liminf _{n \rightarrow \infty} \frac{\omega_{0}(x)+\cdots+\omega_{n}(x)}{n+1} \text { is summable, hence finite a.e. }
$$

In fact, the integral of this function is

$$
\leqq \liminf _{n \rightarrow \infty} \int \frac{\omega_{0}(x)+\cdots+\omega_{n}(x)}{n+1} d m(x)=m X<\infty .
$$

A fortiori,

$$
\omega_{*}(x)=\liminf _{n \rightarrow \infty} \omega_{n}(x)<\infty \text { a.e. }
$$

2.2. We recall that all measures referred to are understood to be $\sigma$-finite and equivalent to $m$.

(1) If there exists an invariant measure $\mu$, and $f$ denotes the Radon-Nikodym derivative $d \mu / d m$, then

$$
\omega_{i}(x)=f(x) / f\left(T^{i} x\right) \text { a.e. } \quad(i=0, \pm 1, \pm 2, \ldots) .
$$

(2) A necessary and sufficient condition for the existence of an invariant measure $\mu$ is the existence of a measurable function $f$ on $X$ such that $0<f(x)<\infty$ and $f(x)$ $=f(T x) \omega(x)$ a.e. $\left({ }^{8}\right)$.

For if $\mu$ exists, (1) (with $i=1$ ) provides such an $f$; conversely, if $f$ exists, we put $\mu(A)=\int_{A} f(x) d m(x)$ for all measurable $A \subset X$.

(3) If $\omega^{*}(x)<\infty$ a.e., there exists an invariant measure.

For, in the notation of 1.7, we may restrict attention to $X_{1}$, since on $X_{2}$ (where $T$ is purely dissipative) there is trivially an invariant measure. Thus by $1.7(1)$ we may assume $\omega^{*}(x)>0$ a.e. From 2.1(1) we obtain $\omega^{*}(x)=\omega^{*}(T x) \omega(x)$, and the result follows from (2).

Similarly, in view of 2.1(4), we have

(4) If $\omega_{*}(x)>0$ a.e., there exists a finite invariant measure $\left({ }^{9}\right)$.

3. Finite invariant measure.

3.1. As one would expect, the question of when there exists a finite invariant measure is much easier to answer than the more general question of the existence of a $\sigma$-finite one. Before stating the theorem, we need a definition. A measure $\mu$ on $X$ is invariantly $\sigma$-finite if $X$ is the union of measurable sets $A_{n}(n=1,2, \ldots)$, each invariant under $T$, and such that $\mu\left(A_{n}\right)<\infty$.

3.2. THEOREM 1. Under the standing hypothesis, the following statements are all mutually equivalent $\left({ }^{10}\right)$.

$\left.{ }^{8}\right)$ [7, p. 571]. The equality $f(x)=f(T x) \omega(x)$ can be replaced by either inequality $(\leqq, \geqq)$; see [12, Lemma 5.3].

$\left({ }^{9}\right)$ This is implicit in [4, Theorem 1].

$\left({ }^{10}\right)$ The equivalence of $(a),(b),\left(b^{\prime}\right)$ also follows from [2, Theorem 5]; see also [5, Theorem 2] and [4, Theorem 1]. It would be interesting to know whether, in ( $\left.b^{\prime}\right)$, the lim inf can be replaced by the lim sup; this question is related to that raised in $3.9(8)$ below. 
(a) $X$ has a finite invariant measure $\mu$ equivalent to $m$.

(b) For almost all $x \in X$,

$$
\lim _{n \rightarrow \infty}\left(\omega_{0}(x)+\cdots+\omega_{n}(x)\right) /(n+1)
$$

exists, say $=l(x)$, and $0<l(x)<\infty$.

(b') For almost all $x \in X$,

$$
0<\liminf _{n \rightarrow \infty}\left(\omega_{0}(x)+\cdots+\omega_{n}(x)\right) /(n+1) .
$$

(c) For almost all $x \in X$, there exists $\alpha_{x}>0$ such that $d\left(\alpha_{x}, 0 ; x\right)>0$.

(d) For almost all $x \in X, I_{0}(x)>0$.

(e) There exists an invariant measure, equivalent to $m$, which is invariantly $\sigma$-finite.

(f) There exists an invariant measure $\nu$, equivalent to $m$, and a nonnegative $\nu$-summable function $h$ on $X$, such that

$$
\lim _{n \rightarrow \infty}\left(\sum_{i=0}^{n} h\left(T^{i} x\right)\right) /(n+1)>0 \text { a.e. }
$$

Further, if these conditions hold, then $l(x)=I_{0}(x)$ a.e., and one finite invariant measure equivalent to $m$ is given by: $\mu(A)=\int_{A} l(x) d m(x)=\int_{A} I_{0}(x) d m(x)$ (for all measurable $A \subset X)\left({ }^{11}\right)$.

The pattern of proof is: (a) $\rightarrow\left(\right.$ b) $\rightarrow\left(b^{\prime}\right) \rightarrow(f) \rightarrow(a),(a) \rightarrow$ (c) $\rightarrow$ (d) $\rightarrow$ (c) $\rightarrow\left(b^{\prime}\right)$, and $(a) \rightarrow(e) \rightarrow(a)$. Since many of these implications are essentially known, we merely sketch their proofs.

3.3. (a) $\rightarrow$ (b). Assume (a); then $T$ is incompressible. Apply the Birkhoff ergodic theorem to the Radon-Nikodym derivative $f=d m / d \mu$ (which is summable because $m X<\infty)$; this gives (b), in view of 2.2(1) (applied to the reciprocal of $f$ ).

3.4. The implication (b) $\rightarrow\left(b^{\prime}\right)$ is trivial. To show $\left(b^{\prime}\right) \rightarrow(f)$, let $g(x)$ denote the lim inf in ( $\left.\mathrm{b}^{\prime}\right)$; put $\nu A=\int_{A} g(x) d m(x), h(x)=1 / g(x)$. Then $\nu$ is finite (2.1(3)), and $(f)$ is satisfied (even with $h>0$ ).

3.5. (f) $\rightarrow$ (a). Assuming (f), let $H(x)$ denote the limit in (f), and put $\mu A$ $=\int_{A} H(x) d \nu(x) ; \mu$ is a finite invariant measure equivalent to $m$.

3.6. (a) $\rightarrow$ (c). Let $\mu$ be a finite invariant measure equivalent to $m$, and put $\phi(x)=d m(x) / d \mu(x), S^{k}=\{x \mid \phi(x) \geqq 1 / k\} ;$ then $\bigcup\left\{S^{k} \mid k=1,2, \ldots\right\}=X$ a.e. Further, write (for each $k=1,2, \ldots) g^{k}=$ characteristic function of $S^{k}$, and

$$
G^{k}(x)=\lim _{n \rightarrow \infty}\left(\sum_{i=0}^{n} g^{k}\left(T^{i} x\right)\right) /(n+1)
$$

which exists finitely a.e. because $\mu\left(S^{k}\right)<\infty$. Let $U_{k}=\left\{x \mid G^{k}(x) \neq 0\right\}$, an invariant set; and put $W_{k}=S^{k}-U_{k}$; we show that $W_{k}$ is null.

${ }^{(11)}$ If $\omega_{*}(x)>0$ a.e., another equivalent finite invariant measure would be given by $\int_{A} \omega_{*}(x) d m(x)$; but this is smaller than $\mu$, in general. 
In fact, consider $\bar{W}_{k}=\bigcup\left\{T^{i} W_{k} \mid i=0, \pm 1, \pm 2, \ldots\right\}$; because $U_{k}$ is invariant and disjoint from $W_{k}$, it is disjoint from $\bar{W}_{k}$ also, and therefore $W_{k} \subset \bar{W}_{k} \cap S^{k}$. Thus

$$
\mu\left(W_{k}\right) \leqq \mu\left(\bar{W}_{k} \cap S^{k}\right)=\int_{W_{k}} g^{k} d \mu=\int_{W_{k}} G^{k} d \mu=0 .
$$

It follows that, disregarding null sets, we have $S^{k} \subset U_{k}$ and thus $X=\bigcup_{k} U_{k}$. For each $x \in X$, choose $k=k(x)$ so that $x \in U_{k}$ : then

$$
\lim _{n \rightarrow \infty}\left(\sum_{i=0}^{n} g^{k}\left(T^{i} x\right)\right) /(n+1)>0 .
$$

But $\sum_{i=0}^{n} g^{k}\left(T^{i} x\right)=$ number of $i$ 's $\leqq n$ for which $\phi\left(T^{i} x\right) \geqq 1 / k$, = number of $i$ 's $\leqq n$ for which $\omega_{i}(x) \geqq 1 /(k \phi(x))$, from $2.2(1)$. Thus we have proved $d(1 /(k \phi(x)), 0 ; x)>0$ a.e.

3.7. The equivalence of (c) and (d) is trivial, because $d(\alpha, 0 ; x) \geqq 0$ and is monotone in $\alpha$. It is easy to see that (c) implies ( $\left.\mathrm{b}^{\prime}\right)$; in fact, assuming (c), we have that for each $x \in X$,

$$
\liminf _{n \rightarrow \infty}\left(\omega_{0}(x)+\cdots+\omega_{n}(x)\right) /(n+1) \geqq \alpha_{x} d\left(\alpha_{x}, 0 ; x\right)>0 .
$$

The implication (a) $\rightarrow$ (e) is trivial, and (e) $\rightarrow$ (a) nearly so; hence all the statements (a)-(f) are equivalent.

3.8. We now assume that (a)-(f) hold, and show first that $l(x)=I_{0}(x)$ a.e.

Let $\mu$ be the equivalent finite invariant measure, and let $f(x)=d m(x) / d \mu(x)$. For each $\alpha \geqq 0$ we write $S_{\alpha}=\{x \mid f(x) \geqq \alpha\}, g_{\alpha}=$ characteristic function of $S_{\alpha}$. From 2.2(1) we have

and therefore

$$
\left\{i \mid i \geqq 0, \omega_{i} \geqq \alpha / f(x)\right\}=\left\{i \mid i \geqq 0, f\left(T^{i} x\right) \geqq \alpha\right\}
$$

$$
d(\alpha / f(x), 0 ; x)=\lim _{n \rightarrow \infty}\left(\sum_{i=0}^{n} g_{\alpha}\left(T^{i} x\right)\right) /(n+1) .
$$

From the ergodic theorem (applied to the $\mu$-summable function $g_{\alpha}$ ), it follows that and therefore

$$
\int_{X} d(\alpha / f(x), 0 ; x) d \mu(x)=\int_{X} g_{\alpha}(x) d \mu(x)=\mu\left(S_{\alpha}\right),
$$

$$
\int_{0}^{\infty}\left\{\int_{X} d(\alpha / f(x), 0 ; x) d \mu(x)\right\} d \alpha=\int_{0}^{\infty} \mu\left(S_{\alpha}\right) d \alpha=\int_{X} f(x) d \mu(x) ;
$$

whence

that is,

$$
\int_{X}\left\{\int_{0}^{\infty} d(\alpha / f(x), 0 ; x) d \alpha\right\} d \mu(x)=m(X)
$$

(1) $\int_{X} I_{0}(x) d m(x)=m(X)$.

In proving 2.1(3) we have shown

(2) $\int_{X} l(x) d m(x) \leqq m(X)$.

But 1.4(3) gives

(3) $l(x) \geqq I_{0}(x)$;

and together (1), (2), (3) prove that $l(x)=I_{0}(x)$ a.e. 
In 3.4 we have implicitly checked that, by defining $\mu A=\int_{A} l(x) d m(x)$, one obtains a finite invariant measure equivalent to $m$; and the proof is complete.

3.9. Remarks and corollaries. (1) The statements (a), (e), (f) of Theorem 1 remain equivalent even if the hypothesis that $m X<\infty$ is dropped (of course $m$ is to be $\sigma$-finite). For they depend only on the equivalence class of $m$.

(2) In (c), $\alpha_{x}$ is necessarily $\leqq \omega^{*}(x)$; and if the assertion holds for one $\alpha_{x}>0$, it also holds for every $\alpha_{x}^{\prime}$ such that $0<\alpha_{x}^{\prime}<\omega^{*}(x)$ (from 1.7(2)).

(3) If (e) holds, then every ( $\sigma$-finite) invariant measure equivalent to $m$ is invariantly $\sigma$-finite.

For let $\nu$ be any such measure; by Theorem 1 there is also a finite invariant measure $\mu$; let $g(x)=d \nu(x) / d \mu(x)$. Then $g$ is invariant, because $\mu$ and $\nu$ are. Write

$$
X_{n}=\{x \mid g(x)<n\} ; \text { then } X=\bigcup\left\{X_{n} \mid n=1,2, \ldots\right\},
$$

each $X_{n}$ is invariant, and $\nu\left(X_{n}\right)=\int_{X_{n}} g(x) d \mu(x)<n \mu\left(X_{n}\right)<\infty$.

(4) Whether or not there is an invariant measure,

$$
\liminf _{n \rightarrow \infty} \frac{\omega_{0}(x)+\cdots+\omega_{n}(x)}{n+1}=I_{0}(x) \text { a.e. }
$$

For there is (modulo null sets) a largest invariant set $Y_{1}$ which admits an invariantly $\sigma$-finite measure (equivalent to $m$ ); and Theorem 1 shows that there is a finite invariant measure on $Y_{1}$, and that

$$
\lim _{n \rightarrow \infty}\left(\omega_{0}(x)+\cdots+\omega_{n}(x)\right) /(n+1)=I_{0}(x) \text { a.e. on } Y_{1} \text {. }
$$

Apart from null sets, no invariant subset of $X-Y_{1}$ can have a finite invariant measure equivalent to $m$; hence, by Theorem 1 again, we must have

$$
\liminf _{n \rightarrow \infty}\left(\omega_{0}(x)+\cdots+\omega_{n}(x)\right) /(n+1)=0 \text { a.e. on } X-Y_{1} \text {. }
$$

From 1.3(3), it follows that $I_{0}(x)=0$ also on $X-Y_{1}$; and the result follows.

From 2.1(3) and (4) we also have that

(5) $I_{0}(x)$ is summable, and hence finite a.e.

(6) We have seen in (4) that there is always a largest invariant subset $Y_{1}$ of $X$ (modulo null sets) which admits a finite invariant measure (equivalent to $m$ ), and that $Y_{1}$ can be characterised as

$$
\begin{aligned}
\left\{x \mid \lim _{n \rightarrow \infty}\left(\omega_{0}(x)+\cdots+\omega_{n}(x)\right) /(n+1)>0\right\} & \\
& =\left\{x \mid \liminf _{n \rightarrow \infty}\left(\omega_{0}(x)+\cdots+\omega_{n}(x)\right) /(n+1)>0\right\}=\left\{x \mid I_{0}(x)>0\right\} .
\end{aligned}
$$

It is not hard to see that $X-Y_{1}$ is characterized (to within a null set) as

$$
\{x \mid d(\alpha, 0 ; x)=0 \text { for all } \alpha>0\} .
$$

(7) If we define $\mu(A)=\int_{A} I_{0}(x) d m(x)$ for all measurable $A \subset X$, the preceding considerations show that $\mu$ is always a finite invariant measure, equivalent to $m$ on 
$Y_{1}$, and identically 0 on $X-Y_{1}$. Further, 3.8(1) gives $\mu(X)=\mu\left(Y_{1}\right)=m\left(Y_{1}\right)$. Thus another necessary and sufficient condition for the existence of a finite invariant measure on $X$, equivalent to $m$, is $\int_{X} I_{0}(x) d m(x)=m(X)$.

(8) If there exists an invariant measure on $X$, equivalent to $m$ and $\sigma$-finite (but not necessarily finite), then it is an easy consequence of the ergodic theorem and the finiteness of $m X$ that $\lim _{n \rightarrow \infty}\left(\omega_{0}(x)+\cdots+\omega_{n}(x)\right) /(n+1)$ exists (and is finite) a.e. The converse seems to be unknown.

(9) For further properties equivalent to those in Theorem 1, see 4.10 below.

\section{Invariant measure; necessary condition.}

4.1. Our main goal in this section is Theorem 4 (4.7), giving a necessary condition for the existence of a possibly infinite (but $\sigma$-finite) invariant measure. Later (Theorem 7, 5.1) we shall prove the converse. As by-products of the methods used to prove Theorem 4, we also obtain some further results which, while not directly relevant, may be of independent interest. In particular, the notion of a "stable $(C, 1)$ limit" $(4.4)$ provides a sharpening of the usual ergodic theorem, and the behavior of $d(\alpha, \beta ; x)$ as $\alpha \rightarrow \infty$ or 0 is determined (Theorems 5 and 6 (4.8, 4.9)).

4,2. First we dispose of the case in which $T$ is dissipative.

THEOREM 2. If $T$ is purely dissipative, then $I_{1}(x)<\infty$ a.e.

We have $(1.7(1)) \omega^{*}(x)=0$; further, $\omega_{0}(x)=1$. Thus the argument proving 1.3(3) applies with $\beta=1$, giving

from [12, Corollary 8.3].

$$
I_{1}(x)=\frac{1}{c} \sum_{i=0}^{\infty} \omega_{i}(x)<\infty
$$

REMARK. If we write $\lambda(x)=\sup \left\{\omega_{n}(x) \mid n \in \mathscr{N}\right\}=\max \left\{\omega_{n}(x) \mid n \in \mathscr{N}\right\}$ here, so that $1 \leqq \lambda(x)<\infty$, the proof of $1.3(3)$ shows that $0<d(\alpha, \beta ; x)<\infty$ whenever $0<\alpha, \beta \leqq \lambda(x)$, and that $0<I_{\beta}(x)<\infty$ whenever $0<\beta<\lambda(x)$. Of course we have $d(\alpha, 0 ; x)=0$ here if $\alpha>0$ (compare Theorem $1,3.2$ ). Note that in any case $I_{\beta}(x)>0$ a.e. if $\beta>0(1.2(7))$.

4.3. In the following theorem and corollaries we relax the standing hypothesis that the measure on $X$ is finite, allowing it to be merely $\sigma$-finite; to emphasise this we denote it by $\mu$ instead of $m$. Before stating the theorem, we need a definition. A real-valued function $f$, defined on an invariant subset $U$ of $X$, will be said to be invariantly $\sigma$-summable on $U$ (with respect to $T$ ) provided $U$ can be written as $\bigcup\left\{V_{n} \mid n=1,2, \ldots\right\}$ where each $V_{n}$ is invariant and $f$ is summable on $V_{n}$. Given an arbitrary measurable real function $f$ on $X$, it is easy to see that there exists (modulo null sets) a largest invariant set $U$ (possibly empty) on which $f$ is invariantly $\sigma$-summable.

THEOREM 3. If $T$ is incompressible and measure-preserving, and $f$ is a positive real-valued measurable function on $X$ such that $\mu\{x \mid f(x) \geqq \alpha\}<\infty$ whenever $\alpha>0$, 
then the largest invariant set $U$ on which $f$ is invariantly $\sigma$-summable is, modulo null sets, precisely $\left\{x \mid J_{\beta}(x)<\infty\right.$ for some positive $\beta$ (depending on $\left.\left.x\right)\right\}$. Further, we have $\lim _{n \rightarrow \infty} \sum_{i=0}^{n} f\left(T^{i} x\right) /(n+1)=J_{0}(x)$ for almost all $x \in U\left({ }^{12}\right)$.

Proof. We begin by deriving some formulae. Writing $S_{\beta}=\{x \mid f(x) \geqq \beta\}$, as usual, let $\bar{S}_{\beta}$ denote the "invariant closure" of $S_{\beta}$ (i.e., the smallest invariant set, modulo null sets, containing $S_{\beta}$ ). Since $T$ is incompressible, we have (as in $[13,4.3]$ ):

$$
\bar{S}_{\beta}=\bigcap_{n} \bigcup_{i} T^{-(n+i)} S_{\beta} \quad(i, n=1,2, \ldots)
$$

Keeping $\beta>0$ fixed for the present, let $V$ be any invariant subset of $\bar{S}_{\beta}$. For arbitrary $\alpha>0$, let $g=$ characteristic function $\chi\left(S_{\alpha} \cap V\right), h=\chi\left(S_{\beta} \cap V\right)$; note that both are summable, and that (from (1)) $h$ is "invariantly positive" on $V$ in the sense of [6, p. 157], so that the Halmos-Hopf ergodic theorem applies to the measure space $(V, \mu)$, the transformation $T \mid V$, and the functions $g$ and $h$. Since we have in fact

$$
\lim _{n \rightarrow \infty} \sum_{0}^{n} g\left(T^{i} x\right) / \sum_{0}^{n} h\left(T^{i} x\right)=D(\alpha, \beta ; x) \quad(x \in V),
$$

it follows from $[6$, p. 160] that

that is,

$$
\int_{V} h(x) D(\alpha, \beta ; x) d \mu(x)=\int_{V} g(x) d \mu(x)
$$

$$
\int_{V \cap S_{\beta}} D(\alpha, \beta ; x) d \mu(x)=\mu\left(S_{\alpha} \cap V\right) .
$$

Integrating with respect to $\alpha$ (and inverting the order of integration on the left), we obtain

$$
\int_{V \cap S_{\beta}} J_{\beta}(x) d \mu(x)=\int_{0}^{\infty} \mu\left(S_{\alpha} \cap V\right) d \alpha=\int_{V} f(x) d \mu(x) .
$$

Now we prove that, for almost all $x \in U, J_{\beta}(x)<\infty$ for some $\beta=\beta(x)>0$. We have $U=\bigcup\left\{U_{n} \mid n \in \mathscr{N}\right\}$ where $U_{n}$ is invariant and $f$ is summable on $U_{n}$. Fixing $n$ and $\beta$ for the moment, put $V=\bar{S}_{\beta} \cap U_{n} \cap\left\{x \mid J_{\beta}(x)=\infty\right\}$; this is invariant because $J_{\beta}$ is (from Theorem B(ii), 1.5), and (3) above applies. The integral on the right is known to be finite; hence so is the integral on the left, and we must have $\mu\left(V \cap S_{\beta}\right)=0$. This works for all $n$, showing that $J_{\beta}(x)<\infty$ for almost all $x \in S_{\beta} \cap U$. But $X=\bigcup\left\{S_{1 / n} \mid n=1,2, \ldots\right\}$; hence, for almost all $x \in U$, we have $J_{1 / n}(x)<\infty$ for some $n$, as required.

Conversely, write $V_{k}=\bar{S}_{\beta} \cap\left\{x \mid J_{\beta}(x)<k\right\}$ where $\beta=1 / k(k=1,2, \ldots)$; because of the monotoneity of $S_{\beta}$ and $J_{\beta}(1.2(9))$ it will suffice to prove $\bigcup_{k} V_{k} \subset U$, and we do this by showing that $f$ is summable on each $V_{k}$. In fact, (3) above applies to

$\left({ }^{12}\right)$ The last assertion is similar to [14, Theorem 1]. 
$V=V_{k}$; now the integral on the left is $\leqq k \mu\left(S_{\beta}\right)<\infty$, so the integral on the right is finite too, as required.

For the remaining assertion of the theorem, it will suffice to consider the sets $U_{n}$ (occurring above) separately; thus we may assume that $f$ is summable on $X$. By the Birkhoff ergodic theorem, $\lim _{n \rightarrow \infty} \sum_{i=0}^{n} f\left(T^{i} x\right) /(n+1)$ exists (finitely) a.e.; we denote it by $f(x)$. Also (Theorem $\mathrm{A}, 1.5)$ the sequence $\left\{f\left(T^{i} x\right) \mid i \in \mathscr{N}\right\}$ is a density sequence, and $1.4(3)$ gives

$$
f(x) \geqq J_{0}(x) \geqq 0 \text { a.e. }
$$

On the invariant set $\{x \mid f(x)=0\}$ we therefore have equality in (4), so we may restrict attention to the complementary invariant set; that is, we may assume $f(x)>0$ for all $x \in X$. By 3.9(1) and 3.9(3), the measure $\mu$ is invariantly $\sigma$-finite; thus we may assume $\mu X<\infty$. We may therefore take $\beta=0$ and $V=X$ in the reasoning leading to (3) above, which now gives $\int_{X} J_{0}(x) d \mu(x)=\int_{X} f(x) d \mu(x)$; but the latter integral is finite, and by the Birkhoff theorem it equals $\int_{X} f(x) d \mu(x)$. Because of (4) it follows that $f(x)=J_{0}(x)$ a.e., as required.

4.4. CoROllary 1. If T is measure-preserving (the measure $\mu$ need not be finite) and $f$ is an arbitrary invariantly $\sigma$-summable function, the sequence $\left\{f\left(T^{n} x\right) \mid n \in \mathscr{N}\right\}$ has a.e. a stable finite $(C, 1)$ limit.

Here a sequence $\xi=\{\xi(n) \mid n \in \mathscr{N}\}$ is said to have a stable $(C, 1)$ limit providing, for every $\mathscr{M} \subset \mathscr{N}$ of asymptotic density 0 , we have that

$$
\lim _{n \rightarrow \infty} \frac{1}{n+1} \sum\{\xi(i) \mid 0 \leqq i \leqq n, i \notin \mathscr{M}\}
$$

exists (possibly infinite); it is then independent of $\mathscr{M}$ (and so, in particular, equals the usual $(C, 1)$ limit). Note that here the denominator $n+1$ could be replaced by the number of $i$ 's such that $0 \leqq i \leqq n$ and $i \notin \mathscr{M}$ without changing the meaning; in other words, $\xi$ has a stable $(C, 1)$ limit if and only if it has a $(C, 1)$ limit and continues to have one (necessarily the same) after the deletion of an arbitrary subsequence, of asymptotic density zero, of its terms. Thus every convergent sequence has a stable $(C, 1) \operatorname{limit}\left({ }^{13}\right)$.

In proving the corollary, we may as usual assume that $f$ is summable; also we may assume $T$ incompressible, since if $T$ is purely dissipative it is well known (cf. $\left[13\right.$, p. 241, (2)]) that $f\left(T^{n} x\right) \rightarrow 0$ a.e. as $n \rightarrow \infty$. First suppose $f(x)>0$ for all $x \in X$. Then Theorem 3 applies, giving $\lim _{n \rightarrow \infty} \sum\{\xi(i) \mid 0 \leqq i \leqq n\} /(n+1)=J_{0}(x)$, where $\xi(i)=f\left(T^{i} x\right)$. The effect here of omitting the summands for which $i \in \mathscr{M}$ is that of replacing the density sequence $\xi$ by the sequence $\eta$, where $\eta(i)=\xi(i)$ if $i \notin \mathscr{M}$, and

$\left({ }^{13}\right)$ It is easy to give examples of a sequence with a finite $(C, 1)$ limit which is not stable, and of a sequence with a finite stable $(C, 1)$ limit which is not convergent. The argument which follows shows that every density sequence $\xi$ which has a $(C, 1)$ limit equal to. $A_{0}(\xi)$ has a stable $(C, 1)$ limit. 
$\eta(i)=0$ if $i \in \mathscr{M}$. From $1.4(1), \eta$ is a weak density sequence, and $A_{0}(\eta)=A_{0}(\xi)$. Thus, from 1.4(3), we have

$$
\liminf _{n \rightarrow \infty} \sum\{\xi(i) \mid 0 \leqq i \leqq n, i \notin \mathscr{M}\} /(n+1) \geqq A_{0}(\eta)=A_{0}(\xi)=J_{0}(x) .
$$

But clearly this lim inf $\leqq$ the corresponding lim sup

$$
\leqq \limsup _{n \rightarrow \infty} \sum\{\xi(i) \mid 0 \leqq i \leqq n\} /(n+1)=J_{0}(x) \text {, and the result follows. }
$$

If we have merely $f(x) \geqq 0(x \in X)$, it is easy to construct a positive summable function $g$; the preceding applies both to $f+g$ and $g$, and it follows for $f$ by subtraction. Finally, in the general case, we can write $f=f^{+}-f^{-}$where $f^{+}$and $f^{-}$are nonnegative summable functions; the assertion of the corollary holds for $f^{+}$and $f^{-}$, and hence for $f$.

4.5. COROLlaRY 2. If $T$ is ergodic, incompressible and measure-preserving (where the measure $\mu$ is not required to be finite), and if $f$ is a positive measurable function on $X$, then $f$ is summable if and only if both the following conditions are satisfied:

(i) for each $\alpha>0, \mu\left(S_{\alpha}\right)<\infty$, where $S_{\alpha}=\{x \mid f(x) \geqq \alpha\}$,

(ii) there exists $\beta>0$ such that $J_{\beta}(x)<\infty$ a.e.

Further, if (i) holds, we have

(iii) $D(\alpha, \beta ; x)=\mu\left(S_{\alpha}\right) / \mu\left(S_{\beta}\right)$ a.e., for all $\alpha, \beta \geqq 0$,

(iv) $J_{\beta}(x)=\int_{X} f d \mu / \mu\left(S_{\beta}\right)$ a.e. $(\beta \geqq 0)\left({ }^{14}\right)$.

The first part of this corollary is an immediate consequence of Theorem 3; and the special case of (iii) in which $\alpha, \beta>0$ and $\mu\left(S_{\beta}\right) \neq 0$ follows from 4.3(2) (applied to $\left.X=\bar{S}_{\beta}=V\right)$, since the invariant function $D(\alpha, \beta ; x)$ must now be constant a.e. If $\mu\left(S_{\beta}\right)=0$ (and $\alpha, \beta>0$ ), we reason as follows. By deleting the union of at most countably many null sets of the form $S_{\gamma}$ we can arrange that (for all $\gamma \geqq 0$ ) $S_{\gamma}$ is null if and only if it is empty; and (iii) follows from our conventions about $\delta_{n}(\alpha, \beta)$ (1.1). To deal with the remaining cases, we observe that if $\mu X<\infty$, all the reasoning in 4.3 applies for $\alpha \geqq 0$ and $\beta \geqq 0$, so we may assume $\mu X=\infty$. The Birkhoff ergodic theorem applied to $\chi\left(S_{\alpha}\right)$ then gives $D(\alpha, 0 ; x)=0$ a.e. when $\alpha>0$, as required, from which it follows that $D(0, \beta ; x)=\infty$ a.e. when $\beta>0$, as required. Finally, the case $\alpha=\beta=0$ is trivial from the convention $\infty / \infty=1$.

The deduction of (iv) from 4.3(3) is similar, but simpler.

4.6. Remarks. Both in Theorem 3 and in Corollary 2, the condition that $f$ be positive could be relaxed (with some trouble) to requiring that $f$ be nonnegative.

If $T$ is allowed to be compressible in Theorem 3, then (by using 1.3(3)) we find $U \subset\left\{x \mid J_{\beta}(x)<\infty\right.$ for some $\left.\beta>0\right\}$; but in general there is no longer equality. Take, for example, $X=$ real line with Lebesgue measure, $T x=x+1, f(x)=1 / 2^{n}$ if $n \leqq x$ $<n+1, f(x)=1 /(n+1)$ if $-n-1 \leqq x<-n(n=0,1,2, \ldots)$. It is easily seen that $J_{\beta}(x)<\infty$ for small enough $\beta=\beta(x)$, but that $f$ is not invariantly $\sigma$-summable.

${ }^{(14)}$ Compare [14, Theorem 2]. 
However, Corollary 2 remains valid to some extent, even if $T$ is compressible. In this case, $X$ must be the union of disjoint atomic sets $A_{i}(i=0, \pm 1, \pm 2, \ldots)$ where $T A_{i}=A_{i+1}$; and if we restrict $f$ to be (say) 0 on each $A_{i}$ with $i<0$, the assertion of Corollary 2 then reduces to $1.3(3)$.

4.7. THEOREM 4. Write $\gamma^{*}(x)=(1 / 2) \omega^{*}(x)$ if this is positive and finite, 1 otherwise. If (in addition to the standing hypothesis) there exists an invariant measure (possibly infinite, but $\sigma$-finite and equivalent to $m$ ) on $X$, then for almost all $x \in X$ we have $I_{\gamma^{*}(x)}(x)<\infty$.

As in 1.7, we write $X=X_{1} \cup X_{2}$ where these are disjoint invariant sets, $T \mid X_{1}$ is incompressible and $T \mid X_{2}$ is purely dissipative. For $x \in X_{2}$ we have $\omega^{*}(x)=0$ (1.7(1)) and $I_{1}(x)<\infty$, by Theorem 2 (4.2). Thus it is enough to consider $T \mid X_{1}$; that is, we may assume that $T$ is incompressible, and hence that $\omega^{*}(x)>0$. Let $\mu$ be the invariant measure; we put $f=d m / d \mu$ and apply Theorem 3 , noting that $f$ is summable on $(X, \mu)$. This gives that $J_{\beta}(x)<\infty$ for some positive $\beta=\beta(x)$, for almost all $x$.

We also have $\omega_{n}(x)=f\left(T^{n} x\right) / f(x)$, from 2.2(1), and therefore

$$
d(\alpha, \beta ; x)=D(\alpha f(x), \beta f(x) ; x) \quad(\alpha, \beta \geqq 0)
$$

and hence

$$
I_{\beta}(x)=J_{\beta f(x)}(x) / f(x) .
$$

Thus, by 1.2(9), we have $I_{\gamma}(x)<\infty$ for every positive $\gamma<\beta(x) / f(x)$, and in particular for such a $\gamma<\omega^{*}(x)$. By $1.7(3)$, it follows that $I_{\gamma}(x)<\infty$ for every positive $\gamma<\omega^{*}(x)$, and in particular for $\gamma=\gamma^{*}(x)$.

Of course, in this theorem $\gamma^{*}(x)$ could be replaced by any other positive number which is less than $\omega^{*}(x)$ when $\omega^{*}(x)>0$, and is $\leqq 1$ when $\omega^{*}(x)=0$.

ReMarK. From (2) and 4.5(iv) we see that if $T$ is ergodic, incompressible and has an invariant measure $\mu$, and if

$$
g=d \mu / d m, \quad H(\beta, x)=\left\{x^{\prime} \mid g\left(x^{\prime}\right) \leqq \beta^{-1} g(x)\right\},
$$

then

$$
I_{\beta}(x)=m(X) g(x) / \int_{H(\beta, x)} g(x) d m(x)
$$

This might perhaps provide a method for determining $g$ (and hence $\mu$ ) from the functions $I_{\beta}, \beta \geqq 0$; however, I have been unable to do this, even assuming that an invariant measure exists. The converse of Theorem 4 is proved later by a quite different method.

4.8. The condition arising in Theorem 4, of the finiteness of

$$
I_{\beta}(x)=\int_{0}^{\infty} d(\alpha, \beta ; x) d \alpha,
$$


raises the question of the behavior of $d(\alpha, \beta ; x)$ for small and for large $\alpha$. We discuss this in the next two theorems.

THEOREM 5. $\lim _{\alpha \rightarrow \infty} d(\alpha, \beta ; x)=0$ for all $\beta \geqq 0$.

Proof. First we note that, as an immediate consequence of the definition of $d(\alpha, \beta ; x)$, we have

$$
\text { if } \omega^{*}(x)<\infty \text {, then } d(\alpha, \beta ; x)=0 \text { for all large enough } \alpha ;
$$

so we may assume that $\omega^{*}(x)=\infty$, and thus that $T$ is incompressible. As in [12, pp. 37, 38] we consider the measure-preserving transformation $T^{*}$ on the product space $X^{*}=X \times Y$, where $Y$ is the interval $(0, \infty)$ with Lebesgue measure, and where $T^{*}(x, y)=(T x, y / \omega(x))$. By [12, Theorem 2, p. 40], $T^{*}$ is also incompressible. For all positive $\alpha, \beta, y$ we have $[13,5.1(1)]$

$$
d(\alpha y, \beta y ; x)=D(\alpha, \beta ;(x, y)) \quad(x \in X)
$$

where $D$ now refers to the function $(x, y) \rightarrow 1 / y$ on $X^{*}$.

We write $S_{\gamma}^{*}=\{(x, y) \mid 0<y \leqq 1 / \gamma\}, \quad(\gamma>0)$ and note that the characteristic functions $\chi\left(S_{\alpha}^{*}\right), \chi\left(S_{\beta}^{*}\right)$ are summable; thus, by the same corollary to the HalmosHopf ergodic theorem that we have used before (applied to the invariant closure $\bar{S}_{\beta}^{*}$ of $S_{\beta}^{*}$ ), we have that, if $\alpha>\beta>0$,

$$
\iint_{X \times Y} \chi\left(S_{\alpha}^{*}\right) d m(x) d y=\iint_{X \times Y} \chi\left(S_{\beta}^{*}\right) D(\alpha, \beta ;(x, y)) d m(x) d y .
$$

(In the first instance, the above formula follows with the integrals extended only over $\bar{S}_{\beta}^{*}$, but outside $\bar{S}_{\beta}^{*}$ both integrands are 0 .) That is,

$$
m X / \alpha=\iint_{X \times\left(0, \beta^{-1}\right)} D(\alpha, \beta ;(x, y)) d m(x) d y \quad \text { if } \alpha>\beta>0 .
$$

The integrand here is a nonnegative decreasing function of $\alpha$, hence

$$
\iint_{X \times\left(0, \beta^{-1}\right)} \lim _{\alpha \rightarrow \infty} D(\alpha, \beta ;(x, y)) d m(x) d y=0,
$$

so that $\lim _{\alpha \rightarrow \infty} D(\alpha, \beta ;(x, y))=0$ a.e. on $X \times\left(0, \beta^{-1}\right)$. From (2) this gives

$$
\lim _{\alpha \rightarrow \infty} d(\alpha y, \beta y ; x)=0 \text { a.e. on } X
$$

for almost all positive $y<1 / \beta$. Fixing a suitable $y$, we have $\lim _{\alpha \rightarrow \infty} d(\alpha, \gamma ; x)=0$ a.e. for some $\gamma$ such that $0<\gamma<\omega^{*}(x)(=\infty)$; and, from 1.2(2) and Theorem $\mathrm{D}(\mathrm{i})$ (1.6), it follows that $\lim _{\alpha \rightarrow \infty} \cdot d(\alpha, \beta ; x)=0$ for all $\beta>0$, and hence also when $\beta=0$ (1.2(3)).

4.9. Before stating the next theorem, we recall (3.9(4)) that there is (modulo null sets) a largest subset $Y_{1}$ of $X$ which admits a finite invariant measure (equivalent to $m)$. We write $\lim _{\alpha \rightarrow 0+} d(\alpha, \beta ; x)$ as $d(0+, \beta ; x)$; the limit of course exists (possibly infinite), since $d(\alpha, \beta ; x)$ is monotone in $\alpha$. The following theorem shows, in fact, that the limit is "usually" infinite. 
THEOREM 6. For almost all $x \in X$ and for all $\beta \geqq 0$ we have $d(0+, \beta ; x)=d(0, \beta ; x)$, except precisely when $x \in X-Y_{1}$ and $\beta=0$. Further, $d(0+, \beta ; x)=\infty$, unless $x \in Y_{1}$ and $\beta \leqq \omega^{*}(x)$. It is finite if $x \in Y_{1}$ and $\beta<\omega^{*}(x)$.

Proof. Let $Z$ be the set of all $x \in X$ such that $d(0+, \beta ; x)<\infty$ for some (and hence all) positive $\beta<\omega^{*}(x)$. We begin by proving

(1) $Z \subset Y_{1}$ (modulo null sets).

For suppose not. Since $Z$ is invariant (from Theorem $\mathrm{D}(\mathrm{ii}), 1.6$ ), we can restrict attention to $Z-Y_{1}$; that is, we may assume $Z=X, m X>0$, and that no nonnull invariant subset of $X$ has a finite invariant measure, and we derive a contradiction. Note that, since $\omega^{*}(x)>0$ on $Z, T$ must be incompressible (1.7(1)).

We apply the same argument as in 4.8, but integrate not over all of $X^{*}=X \times Y$, but over an arbitrary $T^{*}$-invariant subset $V^{*}$ of $\bar{S}_{\beta}^{*}$. Then $4.8(3)$ will be replaced by:

(2) $m^{*}\left\{V^{*} \cap\left(X \times\left(0, \alpha^{-1}\right)\right)\right\}=\iint_{V_{\beta}^{*}} D(\alpha, \beta ;(x, y)) d m(x) d y$, if $0<\alpha, \beta<\omega^{*}(x)$,

where $V_{\beta}^{*}$ denotes $V^{*} \cap\left(X \times\left(0, \beta^{-1}\right)\right)$, and where $m^{*}$ is the product measure on $X^{*}$. Here the integrand is a decreasing function of $\alpha$; hence, taking limits as $\alpha \rightarrow 0+$, we obtain

$$
m^{*}\left(V^{*}\right)=\iint_{V_{\beta}^{*}} D(0+, \beta ;(x, y)) d m(x) d y \quad\left(0<\beta<\omega^{*}(x)\right) .
$$

The integrand here is $(4.7(1)) d(0+, \beta y ; x)$, and is by hypothesis finite for $y \leqq 1$, at least. Hence, on writing $U_{k}=\{(x, y) \mid D(0+, \beta ;(x, y)) \leqq k\}(k=1,2, \ldots)$, and $\varepsilon=\min \left(\beta^{-1}, 1\right)$, we have $X \times(0, \varepsilon) \subset S_{\beta}^{*} \cap \bigcup_{k} U_{k} ;$ and therefore $m^{*}\left(S_{\beta}^{*} \cap U_{k}\right)$ $>0$ for some $k$.

The set $U_{k}$ is invariant (Theorem B(ii), 1.6); thus we may take $V^{*}=\bar{S}_{\beta}^{*} \cap U_{k}$ in the foregoing, and have (from the choice of $k$ and from (3))

(4) $0<m^{*}\left(V^{*}\right)<\infty$.

Let $W$ denote the essential projection of $V^{*}$ on $X$ (that is, $W=$ $\left\{x \mid\left\{y \mid(x, y) \in V^{*}\right\}\right.$ is not null $\left.\}\right) ; W$ is invariant under $T$, and $m(W)>0$. For all measurable $A \subset W$, define $\mu A=m^{*}\left(V^{*} \cap(A \times Y)\right)$; then $\mu$ is an invariant measure on $W$, finite and equivalent to $m$ (from (4)). But this contradicts our assumption, and (1) is proved.

From (1) it follows that $d(0+, \beta ; x)=\infty$ (a.e.) on $X-Y_{1}$, if $0<\beta<\omega^{*}(x)$. A fortiori (1.2(3)) we have

(5) $d(0, \beta ; x)=\infty=d(0+, \beta ; x)$ a.e. on $X-Y_{1}$, for all $\beta>0$.

However, when $\beta=0$, we observe that, from Theorem $1(3.2), d(\alpha, 0 ; x)=0$ a.e. on $X-Y_{1}$, for all $\alpha>0$, so that

(6) $d(0+, 0 ; x)=0$ (but $d(0,0 ; x)=1)$ a.e. on $X-Y_{1}$.

Now we turn to $Y_{1}$ and show first that

(7) $d(0+, 0 ; x)=1(=d(0,0 ; x))$ a.e. on $Y_{1}$. 
There is a finite invariant measure $\mu$ on $Y_{1}$, equivalent to $m$. The usual argument now gives (where now $D$ refers to the function $f=d m / d \mu$, and $S_{\gamma}=\{x \mid f(x) \geqq \gamma\}$ ) that, for each invariant set $V$, and for all $\alpha>0$,

$$
\int_{V} \chi\left(S_{\alpha}\right) d \mu=\int_{V} D(\alpha, 0 ; x) d \mu(x)
$$

that is, $\mu\left(S_{\alpha} \cap V\right)=\int_{V} d(\alpha f(x), 0 ; x) d \mu(x)(\alpha>0)$. We make $\alpha \rightarrow 0$ (using the monotoneity and boundedness of $d)$, obtaining

(8) $\mu(V)=\int_{V} d(0+, 0 ; x) d \mu(x)$.

The integrand here is always $\leqq 1$; if we put $V=\{x \mid d(0+, 0 ; x)<1\},(8)$ shows that $\mu(V)=0$, and (7) follows.

Theorem 1 (3.2) shows that, on $Y_{1}$, we have $\omega^{*}(x)>0$ and $1 \geqq d(\beta, 0 ; x)>0$ for some (and hence all) $\beta$ such that $0<\beta<\omega^{*}(x)$. Hence $(1.2(1)) 1 \leqq d(0, \beta ; x)<\infty$ whenever $0<\beta<\omega^{*}(x)$. Now (1.2(2)) $d(\alpha, \beta ; x)=d(\alpha, 0 ; x) d(0, \beta ; x)$, whence

(9) $d(0+, \beta ; x)=d(0, \beta ; x)$ whenever $x \in Y_{1}$ and $0 \leqq \beta<\omega^{*}(x)$.

If $\beta>\omega^{*}(x)>\alpha>0$, we have trivially $d(\alpha, \beta ; x)=\infty$, and consequently

(10) $d(0+, \beta ; x)=d(0, \beta ; x)=\infty\left(\beta>\omega^{*}(x)\right)$.

Finally we consider what happens when $\beta=\omega^{*}(x)$ and $x \in Y_{1}$. There are two $\operatorname{cases}\left({ }^{15}\right)$ :

(a) $d\left(\gamma, \omega^{*}(x) ; x\right)=\infty$ for all $\gamma$ such that $0<\gamma<\omega^{*}(x)$. In this case, we have at once $d\left(0+, \omega^{*}(x) ; x\right)=d\left(0, \omega^{*}(x) ; x\right)=\infty$.

(b) There exists some $\gamma$ such that $0<\gamma<\omega^{*}(x)$ and $d\left(\gamma, \omega^{*}(x) ; x\right)<\infty$. Here an argument similar to the one proving (9) will deduce from (9) that

$$
d\left(0+, \omega^{*}(x) ; x\right)=d\left(0, \omega^{*}(x) ; x\right)<\infty .
$$

4.10. Corollary. $Y_{1}=Z=\{x \mid d(0+, 0 ; x) \neq 0\}=\{x \mid d(0+, 0 ; x)=1\}$.

4.11. REMARK. It can be shown that

$$
d(\alpha-, \beta ; x)=d(\alpha, \beta ; x)=d(\alpha, \beta+; x) \text { a.e. }
$$

whenever $\alpha, \beta>0$, providing that $\beta<\omega^{*}(x)$ where $T$ is incompressible. This can be deduced from 4.5 (iii) by the following roundabout route. As in 4.8 we transform the assertion to one about the case in which $T$ is measure-preserving. If $(X, m)$ is a normal measure space, it can be decomposed (essentially as a product) into invariant fibers on each of which $T$ is both measure-preserving and ergodic (see [10]); we apply 4.5(iii) to each fiber, obtaining the result when $X$ is normal. Finally, in the general case, we set up a "set-isometry" (cf. [8], [13]) between a suitable countably generated subfield of measurable sets of $X$, taken modulo null sets, and the algebra of measurable sets mod null sets of a normal measure space $X^{\prime}$, and show that this enables the assertion to be transferred from $X^{\prime}$ to $X$. It would be desirable to have a direct proof.

$\left.{ }^{15}\right)$ Both cases can actually arise, even when $T$ is ergodic. 


\section{Invariant measure; sufficiency.}

5.1. In this section we prove the main theorem of this paper:

THEOREM 7. If $T$ is a nonsingular measurable transformation of a measure space $(X, m)$, where $m X<\infty$, then the following statements are mutually equivalent:

(1) $X$ has a $\sigma$-finite measure $\mu$, invariant under $T$, and equivalent to $m$.

(2) $I_{\gamma^{*}(x)}(x)<\infty$ a.e., where $\gamma^{*}(x)=\omega^{*}(x) / 2$ if this is positive and finite, and $\gamma^{*}(x)=1$ otherwise.

(3) For some (or, equivalently, all small enough) positive $\gamma(x), I_{\gamma(x)}(x)<\infty$ a.e.

$$
\int_{0}^{\infty} \frac{d \beta}{I_{\beta}(x)}=1 \text { a.e. (or, equivalently, } \neq 0 \text { a.e.). }
$$

We have already seen that (1) implies (2) (Theorem 4, 4.7), and that (2) and (3) are equivalent (as was remarked in 4.7). Also (3) and (4) are equivalent, from 1.3(1). All that remains, then, is to prove that (3) implies (1). The proof which follows is due to Y. Ito, who greatly simplified my original argument.

We may assume

$$
\omega^{*}(x)=\infty \text { for all } x \in X,
$$

since the set where this is false is an invariant set on which, by $2.2(3)$, there is an invariant measure. Also, from (3), 1.2(7) and 1.7(3), we may assume

$$
1 \leqq I_{1}(x)<\infty \quad(x \in X) .
$$

On writing

$$
A_{k}=\left\{x \mid I_{1}(x)<k+1\right\} \quad(k=1,2, \ldots),
$$

we therefore have

Now put

$$
\bigcup_{k} A_{k}=X
$$

$$
B_{k}=\bigcup\left\{T^{i} A_{k} \mid i=0, \pm 1, \pm 2, \ldots\right\},
$$

the invariant closure of $A_{k}$. We first construct an invariant measure on each $B_{k}$, and it will then be easy to combine these measures to give one on $X$.

5.2. Fixing the positive integer $k$ for the present, we write

(1) $f_{k}=$ characteristic function $\chi\left(A_{k}\right)$, and define

(2) $\phi_{k}(x)=\sup _{n \geqq 0} \omega_{n}(x) f_{k}\left(T^{n} x\right) \quad(x \in X)$.

Clearly $\phi_{k}$ is measurable and nonnegative, and $\phi_{k}(x)=0$ whenever $x \in X-B_{k}$. We shall show that

(3) $\phi_{k}(T x) \omega(x)=\phi_{k}(x)$ a.e. For

$$
\begin{aligned}
\phi_{k}(T x) \omega(x) & =\sup _{n \geqq 0} \omega_{n}(T x) \omega(x) f_{k}\left(T^{n+1} x\right) \\
& =\sup _{n \geqq 0} \omega_{n+1}(x) f_{k}\left(T^{n+1} x\right) \quad \text { by } 2.1(1) \\
& \leqq \phi_{k}(x) .
\end{aligned}
$$


But $T$ is incompressible, because of $5.1(1)$; hence (by $[12,5.3])$ equality must hold here almost everywhere.

The incompressibility of $T$ also shows that, for almost all $x \in B_{k}, T^{n} x \in A_{k}$ for some positive integer $n$ (in fact, for infinitely many-cf. 4.3(1)), so

(4) $\phi_{k}(x)>0$ for almost all $x \in B_{k}$.

We next show

(5) $\phi_{k}(x)<\infty$ a.e.

(This is the only step in the whole argument which really uses the assumption about $I_{1}$.)

To prove (5) we need the lemma that, if $n$ is any integer,

(6) $\omega_{n}(x) d\left(\omega_{n}(x), 1 ; x\right)=I_{1}(x) / I_{1}\left(T^{n} x\right)$ a.e.

In fact, 1.6(2) (with $\beta=1$ ) gives

$$
I_{1}\left(T^{n} x\right)=I_{\omega_{n}(x)}(x) / \omega_{n}(x)=d\left(1, \omega_{n}(x) ; x\right) I_{1}(x) / \omega_{n}(x)
$$

(by $1.7(3))$; and by $1.2(1)$ this last expression is $I_{1}(x) /\left(\omega_{n}(x) d\left(\omega_{n}(x), 1 ; x\right)\right)$, and (6) follows.

Now suppose that (5) is false. We choose and fix a point $x$, outside the null set implied by (6), for which $\phi_{k}(x)=\infty$. By definition of $\phi_{k}$, there exist positive integers $n_{1}<n_{2}<\cdots$ such that $\omega_{n_{i}}(x) \rightarrow \infty$ and $T^{n_{i}}(x) \in A_{k}(i=1,2, \ldots)$-that is, $I_{1}\left(T^{n_{i}} x\right)$ $<k+1$. To facilitate printing, we write $\omega_{n_{i}}(x)$ as $w_{i}$, for short. Then (6), applied to $n_{i}$, gives

(7) $w_{i} d\left(w_{i}, 1 ; x\right)>I_{1}(x) /(k+1)$.

Since $\int_{0}^{\infty} d(\alpha, 1 ; x) d \alpha=I_{1}(x)<\infty$, there exists $t>0$ such that $\int_{t}^{\infty} d(\alpha, 1 ; x) d \alpha$ $<I_{1}(x) /(2 k+2)$, and since $w_{i} \rightarrow \infty$ we can take $i$ so large that $w_{i}>2 t$. Then

$$
\frac{I_{1}(x)}{2(k+1)}>\int_{t}^{\infty} d(\alpha, 1 ; x) d \alpha \geqq \int_{w_{1} / 2}^{w_{i}} d(\alpha, 1 ; x) d \alpha \geqq d\left(w_{i}, 1 ; x\right) w_{i} / 2,
$$

in contradiction to (7). Thus (5) is established.

It follows at once (2.2(3)) that there exists an invariant measure ( $\sigma$-finite and equivalent to $m$ ) on $B_{k}$.

5.3. To obtain a suitable invariant measure $\mu$ on all $X$, we note that $A_{1} \subset A_{2} \subset \ldots$ and consequently $B_{1} \subset B_{2} \subset \ldots$. Write $C_{k}=B_{k}-B_{k-1}$ (where $B_{0}=\varnothing$ ); then $C_{1}$, $C_{2}, \ldots$ are disjoint invariant sets, and $\bigcup_{k} C_{k}=X$. Writing

$$
\phi(x)=\sum_{k=1}^{\infty} \chi\left(C_{k} ; x\right) \phi_{k}(x)=\sum_{k=1}^{\infty} \phi_{k}(x)\left(f_{k}(x)-f_{k-1}(x)\right)
$$

we see from 5.2 that $\phi$ is measurable, positive and finite, and satisfies $\phi(T x) \omega(x)$ $=\phi(x)$ a.e. Thus on setting

$$
\mu(A)=\int_{A} \phi(x) d m(x)
$$

we obtain the desired invariant measure. 
5.4. It may be worth stating explicitly what the above construction for $\mu$ comes to in the general case, without making simplifying assumptions about $\omega^{*}$. We decompose $X$ into the disjoint invariant sets $Z_{1}, Z_{2}, Z_{3}$, where

$$
Z_{1}=\left\{x \mid 0<\omega^{*}(x)<\infty\right\}, \quad Z_{2}=\left\{x \mid \omega^{*}(x)=0\right\}, \quad Z_{3}=\left\{x \mid \omega^{*}(x)=\infty\right\} .
$$

For subsets of $Z_{1}$, we put $\mu_{1}(A)=\int_{A} \omega^{*}(x) d m(x)$. For $A \subset Z_{2}$, we note that there is a wandering set $W \subset Z_{2}$ (necessarily an atom) such that

$$
Z_{2}=\bigcup\left\{T^{i} W \mid i=0, \pm 1, \ldots\right\},
$$

and we put $\mu_{2}(A)=$ number of $i$ 's for which $m\left(A \cap T^{i} W\right) \neq 0$. For $A \subset Z_{3}$ we define $\mu_{3}(A)=\int_{A} \phi d m$, where $\phi$ is the function given by 5.3 (applied to $Z_{3}$ ). The desired invariant measure $\mu$ is then of course given by $\mu(A)=\mu_{1}\left(A \cap Z_{1}\right)+\mu_{2}\left(A \cap Z_{2}\right)$ $+\mu_{3}\left(A \cap Z_{3}\right)$.

5.5 It may also be of interest to compare the foregoing decomposition of $X$ with a less familiar one. We suppose the standing hypothesis fulfilled, but do not assume the existence of an invariant measure. In view of 3.9(6) we can express $X$ as the union of disjoint invariant sets $Y_{1}, Y_{2}, Y_{3}$, where $Y_{1}$ is the largest invariant subset of $X$ which admits a finite invariant measure $\left({ }^{16}\right), Y_{1} \cup Y_{2}$ is the largest invariant subset admitting a $\sigma$-finite invariant measure, and $Y_{3}$ is characterized by the fact that no (nonnull) invariant subset of $Y_{3}$ can admit any $\sigma$-finite invariant measure (equivalent to $m$, of course). In Theorem 1 we have seen that $Y_{1}=\left\{x \mid I_{0}(x)>0\right\}$ (cf. also 3.9(6)). It follows from Theorem 7 that $Y_{2}=\left\{x \mid I_{0}(x)=0\right.$ and $I_{\gamma(x)}(x)<\infty$ for some $\gamma(x)>0\}$, and that $Y_{3}=\left\{x \mid I_{y}(x)=\infty\right.$ for all $\left.\gamma>0\right\}$. Further, from 1.3(1) we see that the characteristic function of $Y_{1} \cup Y_{2}$ is precisely $\int_{0}^{\infty}\left(d \beta / I_{\beta}(x)\right)$.

With $Z_{1}, Z_{2}, Z_{3}$ as before (5.4) we see that $Z_{2} \subset Y_{2}$ since $T \mid Z_{2}$ is purely dissipative, and (2.2(3)) $Z_{1} \cup Z_{2} \subset Y_{1} \cup Y_{2}$; thus $Z_{3} \supset Y_{3}$.

From Theorems 1 and 7 we also see that (a.e.) $I_{0}(x)>0$ must imply $I_{\gamma}(x)<\infty$ for all small enough positive $\gamma$. This also follows directly from 1.3(4), in view of 3.9(5).

\section{Extension and application.}

6.1. We have required throughout, in the standing hypothesis, that $m X<\infty$. This, of course, involves no essential loss of generality; but it may be worth while to state explicitly some sample results which follow if the assumption that $m X<\infty$ is dropped. We have a nonsingular measurable (1-1, onto) transformation $T$ of $(X, m)$, where the measure $m$ is merely required to be $\sigma$-finite. A finite equivalent measure is then given by $\int f d m$ where $f$ is any positive summable function. By applying Theorem $\mathrm{C}$ (1.6) to this new measure, we find that the sequence $\left\{f\left(T^{n} x\right) \omega_{n}(x) \mid n \in \mathscr{N}\right\}$ is a.e. a density sequence; and from Theorems 1 and 7 $(3.2,5.1)$ we have (inter alia):

${ }^{(16)}$ As usual, it is understood that we are considering only measurable sets, and disregarding null sets. 
THEOREM 8. A necessary and sufficient condition that there exist an invariant measure on $X$, $\sigma$-finite and equivalent to $\mathrm{m}$, is that for some positive summable function $f$ on $X$ (or, equivalently, for all), and for some positive $\gamma=\gamma(x, f)$ (or, equivalently, for all small enough positive $\gamma\left({ }^{17}\right)$ ) the density average of the sequence $\left\{f\left(T^{n} x\right) \omega_{n}(x) \mid n \in \mathscr{N}\right\}$ with respect to $\gamma$ is finite a.e. A necessary and sufficient condition that there exist a finite invariant measure equivalent to $m$ is that, for some (or, equivalently, every) positive summable function $f$ on $X$, the density average of the above sequence with respect to 0 is positive a.e.

It is a routine matter to translate the rest of Theorems 1 and 7 to the present more general setting.

6.2. Direct sums. As an application of Theorem 7, we show that (under reasonable conditions) a direct sum of measure spaces will have an invariant measure if and only if the summands have invariant measures. We use the following notation. We write $(\tilde{X}, \tilde{m})=\sum\left\{\left(Z_{y}, \lambda_{y}\right) \mid y \in(Y, m)\right\}$ to mean that the measure space $(\tilde{X}, \tilde{m})$ is the direct sum of measure spaces $\left(Z_{y}, \lambda_{y}\right)$ over a measure space $(Y, m)$ (see $[9$, p. 180], for example). As always, we assume all these measures to be $\sigma$-finite.

We say that a measure space is "strictly generated" by a family $\mathscr{A}$ of its measurable subsets if every measurable set is the union of a null set and a member of the Borel field generated by $\mathscr{A}$. We call the above direct sum strict if there exists a countable family $\mathscr{A}=\left\{A_{1}, A_{2}, \ldots\right\}$ of measurable subsets of $\tilde{X}$ which strictly generates $(\tilde{X}, \tilde{m})$ and moreover is such that, for (almost) every $y \in Y$, the family $\left\{A_{n} \cap Z_{y} \mid n \in \mathscr{N}\right\}$ strictly generates $\left(Z_{y}, \lambda_{y}\right)\left({ }^{18}\right)$.

THEOREM 9. Let $(\tilde{X}, \tilde{m})=\sum\left\{\left(Z_{y}, \lambda_{y}\right) \mid y \in(Y, m)\right\}$ be a strict direct sum, and let $T$ be a nonsingular measurable transformation of $\tilde{X}$ under which each $Z_{y}$ is invariant. Then

(i) For almost all $y \in Y, T \mid Z_{y}$ is a nonsingular measurable transformation of $\left(Z_{y}, \lambda_{y}\right)$; and if further $T$ admits an invariant measure $\tilde{\mu}$ (equivalent to $\tilde{m}$ ), then $T \mid Z_{y}$ admits an invariant measure $\mu_{y}$ (equivalent to $\lambda_{y}$ ) such that

$$
(\tilde{X}, \tilde{\mu})=\sum\left\{\left(Z_{y}, \mu_{y}\right) \mid y \in(Y, m)\right\} \text {. }
$$

(ii) Conversely, if each $T \mid Z_{y}$ admits an invariant measure $\mu_{y}$ (equivalent to $\lambda_{y}$ ), then $T$ admits an invariant measure $\tilde{\mu}$ (equivalent to $\tilde{m}$ ).

Sketch of proof. It is easy to see that we can alter $\tilde{m}$ and $\lambda_{y}$, if necessary, to make them finite (without losing the direct sum property). In the following argument, null sets will be repeatedly discarded from $\tilde{X}$; it is easy to see that we can

$\left({ }^{17}\right)$ Or, equivalently, for $\gamma=\frac{1}{2} \lim \sup _{n \rightarrow \infty} f\left(T^{n} x\right) \omega_{n}(x)$ if this is positive and finite, $f(x)$ otherwise.

${ }^{\left({ }^{18}\right)}$ An example of a nonstrict direct sum is: $\tilde{X}=$ plane $R \times R$ and $Y=R$, with usual measures; $Z_{y}=R \times\{y\}$ with $\lambda_{y}$ an inseparable extension of Lebesgue measure. I take this opportunity of pointing out that, as this example shows, Theorem 5 of $[10$, p. 156] is incorrect as stated; the last assertion of that theorem needs modification in general, but becomes correct if the direct sum there is assumed to be strict. 
make them invariant and to consist of the entire summands $Z_{y}$ for a null set of $y$ 's, together with $\lambda_{y}$-null subsets of the remaining $Z_{y}$ 's. Thus, without losing the hypotheses of Theorem 9, we may assume that the Radon-Nikodym derivative $\omega$ of $T$ is everywhere positive and finite, and that $\omega \mid Z_{y}$ is $\lambda_{y}$-measurable and $\lambda_{y}$-summable for all $y \in Y$.

To prove (i), let $A$ be an arbitrary measurable subset of $\tilde{X}$; we assert

$$
\int_{z_{y}} \omega(z) \chi(A ; z) d \lambda_{y}(z)=\lambda_{y}\left(T A \cap Z_{y}\right)
$$

for almost all $y \in Y$. For an easy calculation shows that both sides of (1) have equal integrals over every measurable $B \subset Y$. By discarding countably many null sets, we arrange that (1) holds without exception when $A$ runs over the countable generating system $\mathscr{A}$. Because both sides of (i) are countably additive, it follows that (1) now holds for all $A$ in the Borel field generated by $\mathscr{A}$. Using the fact that the direct sum is strict, we deduce that (1) holds (without exceptions) whenever $A$ is a measurable subset of $\left(Z_{y}, \lambda_{y}\right)$. It follows that, if we write $T_{y}=T \mid Z_{y}$, then $T_{y}$ is a measurable nonsingular transformation of $Z_{y}(y \in Y)$, and its Radon-Nikodym derivative is $\omega \mid Z_{y}$.

Now let $f=d \tilde{\mu} / d \tilde{m}$, and define the measure $\mu_{y}$ on $Z_{y}$ by

$$
\mu_{y}(C)=\int_{C}\left(f \mid Z_{y}\right) d \lambda_{y}
$$

for all measurable subsets $C$ of $Z_{y}$. It is easy to verify that the requirements are satisfied.

In (ii) we have as before (or from the assumptions on $T_{y}$ ) that $T_{y}$ is measurable and nonsingular; and the previous argument shows that the " $\omega$ " for $T_{y}$ is just $\omega \mid Z_{y}$. It follows that the " $\omega_{n}$ " for $T_{y}$ is $\omega_{n} \mid Z_{y}$, and that the " $I_{\gamma}$ " for $T_{y}$ is $I_{\gamma} \mid Z_{y}$. By Theorem 7 this is finite a.e. for small enough $\gamma$; hence, again by Theorem 7, $T$ has an invariant measure $\tilde{\mu}$.

\section{REFERENCES}

1. L. K. Arnold, On $\sigma$-finite invariant measures, Abstract 632-8, Notices Amer. Math. Soc. 13 (1966), 326.

2. A. P. Calderon, Sur les mesures invariantes, C. R. Acad. Sci. Paris 240 (1955), 1960-1962.

3. Y. N. Dowker, Finite and o-finite invariant measures, Ann. of Math. (2) 54 (1951), 596-607.

4. - On measurable transformations in finite measure spaces, Ann. of Math. (2) 62 (1955), 504-516.

5. - Sur les applications mesurables, C. R. Acad. Sci. Paris 242 (1956), 329-331.

6. P. R. Halmos, An ergodic theorem, Proc. Nat. Acad. Sci. U.S.A. 32 (1946), 156-161.

7. - Invariant measures, Ann. of Math. (2) 48 (1947), 735-754.

8. P. R. Halmos and J. von Neumann, Operator methods in classical mechanics. II, Ann. of Math. (2) 43 (1942), 332-350.

9. K. Jacobs, Neuere Methoden und Ergebnisse der Ergodentheorie, Springer, Berlin, 1960. 
10. D. Maharam, Decompositions of measure algebras and spaces, Trans. Amer. Math. Soc. 69 (1950), 142-160.

11. - The representation of abstract integrals, Trans. Amer. Math. Soc. 75 (1953), 154-184.

12. — Incompressible transformations, Fund. Math. 56 (1964), 35-50.

13. - On the Radon-Nikodym derivatives of measurable transformations, Trans. Amer. Math. Soc. 122 (1966), 229-248.

14. F. V. Sirikov, Solution of a problem of Halmos, Uspehi Mat. Nauk 15 (1960), 185-192= Amer. Math. Soc. Transl. (2) 29 (1963), 247-254.

\section{UNIVERSITY OF ROCHESTER,}

ROCHESTER, NEW YORK 